\title{
Bmi-1 cooperates with Foxg1 to maintain neural stem cell self-renewal in the forebrain
}

\author{
Christopher A. Fasano, ${ }^{1,2,7}$ Timothy N. Phoenix ${ }^{1,3}$ Erzsebet Kokovay, ${ }^{1,3}$ Natalia Lowry, ${ }^{1,3}$ \\ Yechiel Elkabetz, ${ }^{2}$ John T. Dimos, ${ }^{4,5}$ Ihor R. Lemischka, ${ }^{4,6}$ Lorenz Studer, ${ }^{2}$ and Sally Temple ${ }^{1,3}$ \\ ${ }^{1}$ Center for Neuropharmacology and Neuroscience, Albany Medical College, Albany, New York 12208 USA; ${ }^{2}$ Developmental \\ Biology Program, Division of Neurosurgery, Sloan-Kettering Institute, New York, New York 10021, USA; ${ }^{3}$ New York Neural \\ Stem Cell Institute, Rensselaer, New York 12144, USA; ${ }^{4}$ Department of Molecular Biology, Princeton University, Princeton, \\ New Jersey 08544, USA; ${ }^{5}$ Izumi Bio, Inc., South San Francisco, California 94080, USA; ${ }^{6}$ Department of Cell and Gene Medicine, \\ Black Family Stem Cell Institute, The Mount Sinai School of Medicine, New York, New York 10029, USA
}

\begin{abstract}
Neural stem cells (NSCs) persist throughout life in two forebrain areas: the subventricular zone (SVZ) and the hippocampus. Why forebrain NSCs self-renew more extensively than those from other regions remains unclear. Prior studies have shown that the polycomb factor Bmi-1 is necessary for NSC self-renewal and that it represses the cell cycle inhibitors p16, p19, and p21. Here we show that overexpression of Bmi-1 enhances self-renewal of forebrain NSCs significantly more than those derived from spinal cord, demonstrating a regional difference in responsiveness. We show that forebrain NSCs require the forebrain-specific transcription factor Foxg1 for Bmi-1dependent self-renewal, and that repression of p21 is a focus of this interaction. Bmi-1 enhancement of NSC selfrenewal is significantly greater with increasing age and passage. Importantly, when Bmi-1 is overexpressed in cultured adult forebrain NSCs, they expand dramatically and continue to make neurons even after multiple passages, when control NSCs have become restricted to glial differentiation. Together these findings demonstrate the importance of Bmi-1 and Foxg1 cooperation to maintenance of NSC multipotency and self-renewal, and establish a useful method for generating abundant forebrain neurons ex vivo, outside the neurogenic niche.
\end{abstract}

[Keywords: Bmil; SVZ; adult stem cells; neural stem cells; self-renewal]

Supplemental material is available at http://www.genesdev.org.

Received September 22, 2008; revised version accepted January 16, 2009.

Neural stem cells (NSCs) are abundant during CNS development and can be isolated from many CNS regions. However, adult mammals retain just two major neural germinal zones, both of which are located in the forebrain: the subgranular zone of the dentate gyrus that gives rise to hippocampal glutamatergic neurons (Kaplan and Hinds 1977; Kaplan and Bell 1984; Gage 2000), and the subventricular zone (SVZ) of the lateral ventricles that produces olfactory bulb neurons (Luskin 1993; Lois and Alvarez-Buylla 1994; Marshall et al. 2003). Why stem cells persist in the forebrain rather than other CNS regions is unclear.

Previous studies have shown that loss of the polycomb protein Bmi-1 in knockout mice depletes postnatal NSCs by inhibiting self-renewal via suppression of the cell cycle inhibitors p16 and p19 (Molofsky et al. 2003; Bruggeman et al. 2007).We further showed by acute gene knockdown

${ }^{7}$ Corresponding author.

E-MAIL fasanoc@mskcc.org; FAX (646) 422-2062.

Article is online at http://www.genesdev.org/cgi/doi/10.1101/gad.1743709. using shRNA that Bmi-1 is also important for NSC proliferation and survival at earlier embryonic stages, partly via suppression of another cell cycle inhibitor, p21 (Fasano et al. 2007).

The broad expression profile of Bmi-1 and these cell cycle inhibitors throughout the nervous system cannot explain why NSCs expand so successfully in the forebrain compared with other CNS regions. Previous studies have shown that forebrain development requires a regionally expressed molecule that also impacts p21 regulation: Foxg1, providing a potential link. Foxg1 is a member of the winged-helix gene family that is expressed in forebrain progenitor cells throughout development and into adulthood (Dou et al. 1999; L. Shen et al. 2006).

Here we examined the role of Bmi-1 and Foxg1 in regional maintenance of NSCs. We show that increasing Bmi-1 expression in forebrain neural progenitor cells (NPCs) (we use the term NPC to indicate all immature proliferating precursor cells, including NSCs) markedly enhances their proliferation and self-renewal. Importantly, even after $1 \mathrm{mo}$ in culture, adult SVZ cells overexpressing 
Bmi-1 continue to generate large numbers of neurons, while in control conditions, neuropotency is rapidly lost over a few passages. Thus, NSCs extracted from the neurogenic niche and placed in culture can maintain multipotency and self-renewal when the level of Bmi-1 is increased. We found that overexpression of Bmi-1 in forebrain NPCs is accompanied by an early and sustained increase in Foxg1. Knockdown or knockout of Foxg1 abolishes the Bmi-1-mediated increase in NSC self-renewal. Foxg1 is not the sole downstream target responsible for the Bmi-1-mediated overexpression phenotype, because increasing Foxg1 levels does not markedly stimulate self-renewal, although it does increase NPC survival. We show that this dramatic increase in NSC self-renewal is region-specific and is not seen after Bmi-1 overexpression in spinal cord progenitors, which do not express Foxg1. Our study demonstrates that Bmi-1 and Foxg1 are both necessary for forebrain NSC self-renewal, offering novel insight into regional stem cell maintenance. We also describe Bmi-1 overexpression as a method for expanding multipotent NSCs and increasing neuron generation from adult SVZ cells in culture.

\section{Results}

Bmi-1 overexpression increases embryonic forebrain NPC self-renewal in vitro and in vivo

Previous studies have shown that knockdown of Bmi-1 prevents NSC self-renewal (Zencak et al. 2005; Fasano et al. 2007). However, gain-of-function studies that examine whether enhancing Bmi-1 will increase NSC selfrenewal have not yet been performed. To explore this issue, forebrain NPCs were isolated at embryonic day 11 (E11), E14, and E16, and postnatal ages P0 and adult. The tissue was dissociated to single cells, which were then transduced with either an empty vector (EV) control lentiviral vector or a lentiviral vector expressing the Bmi-1 ORF that we showed effectively increases Bmi-1 protein levels in NPCs (Bmi-1 Over) (Fasano et al. 2007). The cells were plated in nonadherent conditions, and the resulting floating multicellular neurospheres (an indicator of NSC growth) were counted at $7 \mathrm{~d}$. Primary neurospheres expressing Bmi-1 Over formed at a similar frequency to control neurospheres expressing EV (data not shown). We then dissociated the two sets of primary spheres to single cells and replated these in neurosphere-forming conditions again: The incidence of secondary spheres is a measure of stem cell self-renewal within the original spheres (Reynolds and Weiss 1992).There was a significant increase in secondary neurosphere frequency after Bmi-1 Over compared with EV control, which increased with advancing developmental stages (E11 $155.6 \%$ vs. control; adult $831.9 \%$ vs. control) (Fig. 1A). The enhancement in neurosphere frequency also increased with passage, and at each passage it was most dramatic for adult NSCs (E11 302\% vs. control; adult $3721 \%$ vs. control) (Fig. 1B,C). These findings show that Bmi-1 expression increases the selfrenewal of NSCs and does so most effectively in the adult SVZ population.
To examine whether Bmi-1 also stimulates progenitor proliferation in vivo, Bmi-1 Over or EV control lentiviral vector plasmids were injected into E14 mouse lateral ventricles in utero and electroporated into the developing cerebral cortex, and the results were assessed $3 \mathrm{~d}$ later (Fig. 2). Consistent with the normal pattern of development seen previously with control virus (Bai et al. 2003; Siegenthaler and Miller 2005), the majority of EV control transduced cells migrated into the cortical plate $(\mathrm{CP})$, where they adopted a neuronal morphology, with few cells remaining in the VZ and SVZ germinal zones (Fig. $2 \mathrm{~A}, \mathrm{C}, \mathrm{E})$. However, after $3 \mathrm{~d}$ of Bmi-1 overexpression, there were significantly more $\mathrm{GFP}^{+}$cells retained in the $\mathrm{VZ}$ and SVZ and a significant reduction in the number of cells that had migrated out to the CP (Fig. 2B,D,F; Supplemental Fig. 1A,C).

To assess the fate of the transduced cells, we stained cryostat sections with the progenitor marker Nestin and the proliferative marker Ki67 (Fig. 2C-F). The vast majority of Bmi-1-overexpressing cells were $\mathrm{Ki}^{+} 7^{+}(83 \% \pm$ $11 \%)$ and Nestin $^{+}(71 \% \pm 16 \%)$ compared with $11 \% \pm$ $4 \%$ and $16 \% \pm 5 \%$, respectively, for EV control. Thus, Bmi-1 overexpression in vivo at mid-gestation markedly expands the NPC population and maintains them in a proliferative state, while inhibiting their production of migrating, differentiated neurons over a 3 -d period.

Bmi-1overexpression preserves the normal developmental potential of embryonic cortical NPCs

Bmi-1 overexpression dramatically expands NPCs in neurosphere culture and in vivo, raising the question of whether these cells could still differentiate, and if so, what cell types would be produced. In normal cortical development, cells are born in a specific order: Neurons for deep cortical layers are born before those destined for superficial layers, and after this period of neurogenesis, glial cells are produced (Qian et al. 1997; Q. Shen et al. 2006). Hence we could use these temporal markers to assess the ability of Bmi-1-overexpressing cortical NPCs to differentiate appropriately.

Embryonic cortical neurospheres expressing Bmi-1 Over or EV constructs were plated into adherent conditions in medium without added FGF or EGF, conditions that normally stimulate differentiation. As expected, E11 EV-transduced primary neurospheres differentiated rapidly, producing first neurons then glia over $14 \mathrm{~d}$ of adherent culture. E11 Bmi-1 Over-transduced primary neurospheres, although much larger than their control EV counterparts, also produced differentiated cells on a similar time course. Although the percentage of neurons generated was similar in the two conditions, the actual number of neurons made was significantly higher in the Bmi-1 Over treated spheres, reflecting their increased expansion (Fig. 3A,E).

In the developing dorsal telencephalon in vivo, most of the neurons born at early stages of development are those destined for deep cortical layers, and these can be identified with layer-specific markers, such as the transcription factor ER81, which marks a subpopulation of layer 5 
A

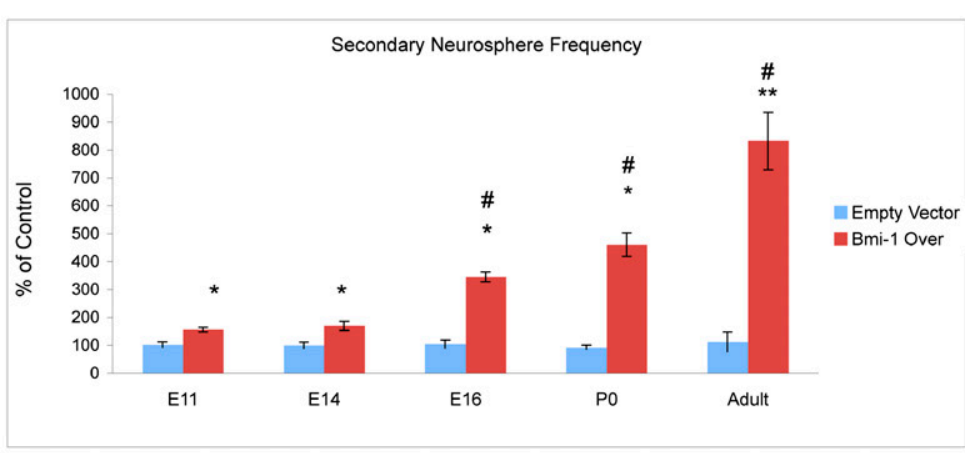

B

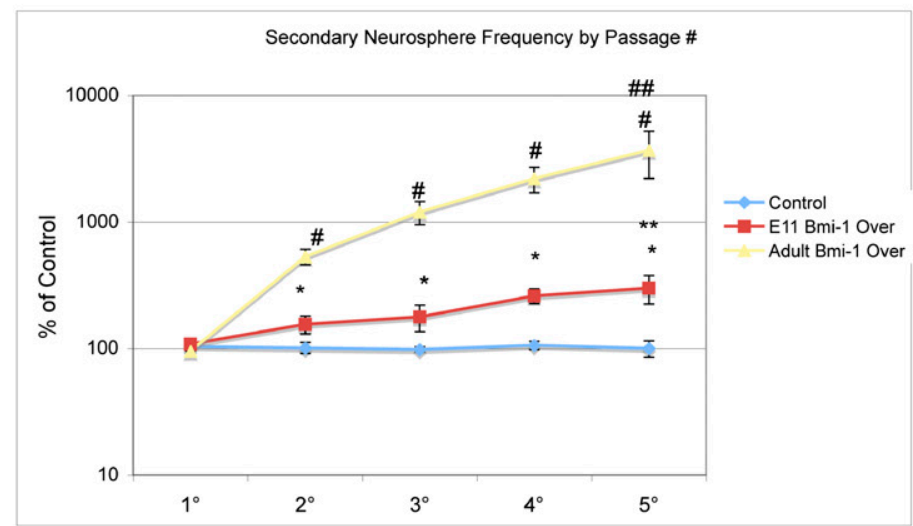

C
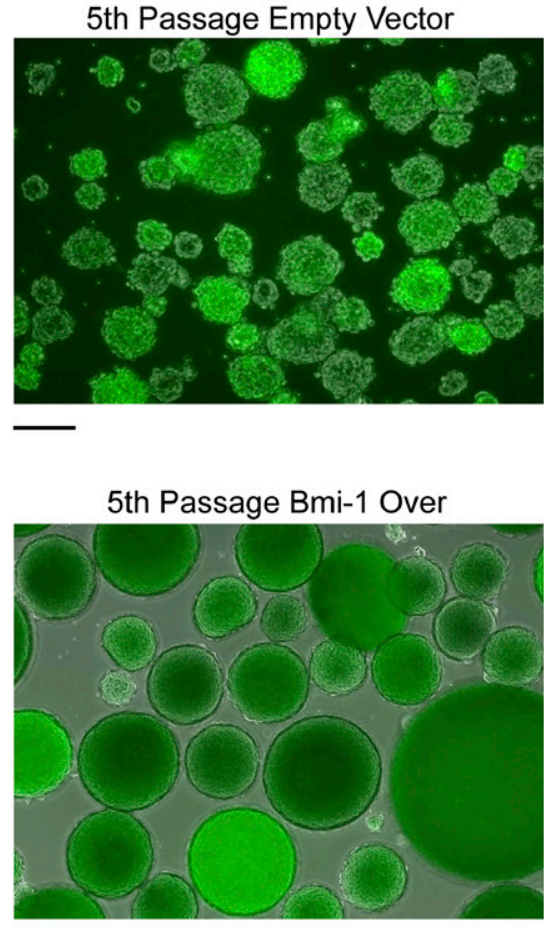

Figure 1. Bmi-1 expression enhances self-renewal of embryonic and adult NSCs in vitro. $(A)$ Bmi-1 overexpression enhances secondary neurosphere frequency increasingly with stage $\left(n=4\right.$ experiments; Bmi-1 Over expression versus EV control: $\left[{ }^{\star}\right] P<0.01$; versus E11 Bmi-1Over: [\#] $\left.P<0.005,\left[{ }^{*}\right] P<0.001\right)$. Values are expressed as percent of control set to $100 \%$. $(B)$ Bmi-1-mediated expansion of NPCs increases with passage and stage $\left(n=4\right.$ experiments; versus control $\left[{ }^{*}\right] P<0.05$, [\#] $P<0.001$; versus $1^{\circ}$ spheres [ $\left.{ }^{* *}\right] P<0.01$, [\#\#] $P<$ 0.001). Values are expressed as percent of control set to $100 \%$. (C) Merged phase/fluorescence micrographs of adult SVZ fifth-passage neurospheres expressing EV control or Bmi-1 Over vectors; transduced cells are visualized by GFP expression (bar, $100 \mu \mathrm{m})$.

neurons. We found that after Bmi-1 overexpression, just as in control, the early-born neurons included ER81 ${ }^{+}$ deep-layer cells, although more were generated after Bmi-1 overexpression $(39 \% \pm 7 \%)$ versus EV control $(24 \% \pm 4 \%)$ (Fig. 3A,E). With subsequent passages, Bmi1-overexpressing cortical neurosphere cells again behaved similarly to control EV neurosphere cells, differentiating largely into later-forming, glial progeny (Fig. 3B$\mathrm{D}, \mathrm{F}-\mathrm{H})$. The generation of oligodendrocyte lineage cells, indicated by staining for the surface marker O4 (Fig. $3 \mathrm{C}, \mathrm{D}, \mathrm{G}, \mathrm{H})$, was enhanced at later passages of cells transduced with Bmi-1 Over compared with EV control (34\% $\pm 7 \%$ vs. $19 \% \pm 4 \%$ ). These results indicate that Bmi- 1 effectively expands the NPC population, but does not prevent them from producing the normal order of CNS cell types when they are stimulated to differentiate.

\section{Bmi-1 overexpression maintains adult SVZ stem cell multipotency, even out of the niche}

The SVZ is the larger of the two postnatal germinal zones (Luskin 1993; Lois and Alvarez-Buylla 1994; Marshall et al. 2003). The rare SVZ stem cells can be prospectively isolated and expanded in culture (Reynolds and Weiss 1992; Capela and Temple 2002) but quickly lose their ability to generate neurons with passaging, and instead produce largely glia. Thus while adult forebrain NSCs offer an alternative to embryonic NSCs for clinical and therapeutic applications, their inefficient neuron production ex vivo and limited differentiation potential are obstacles to their use. Hence, it is important to discover how we can maintain adult SVZ NSC neuropotency outside of the adult neurogenic stem cell niche.

Having shown that Bmi-1 overexpression was able to expand embryonic NPCs and allowed them to produce the cell types they normally produce in vivo, we then assessed whether Bmi-1 overexpression could similarly expand adult SVZ cells and maintain their normal developmental potential-producing neurons. Adult fifthpassage neurospheres transduced with Bmi-1 Over or EV lentiviral vectors were plated into adherent conditions without mitogenic factors (to induce differentiation) for $14 \mathrm{~d}$ and then stained for neuronal and glial markers. EV control transduced fifth-passage neurospheres produced negligible numbers of neurons and few glial cells: Instead, these late-passage control cells were refractory to the differentiation protocol (Fig. 4A-C), and most retained expression of the progenitor marker Nestin (Fig. 4B, inset). In contrast, Bmi-1 Over-expressing adult SVZ fifth-passage neurospheres produced substantial 


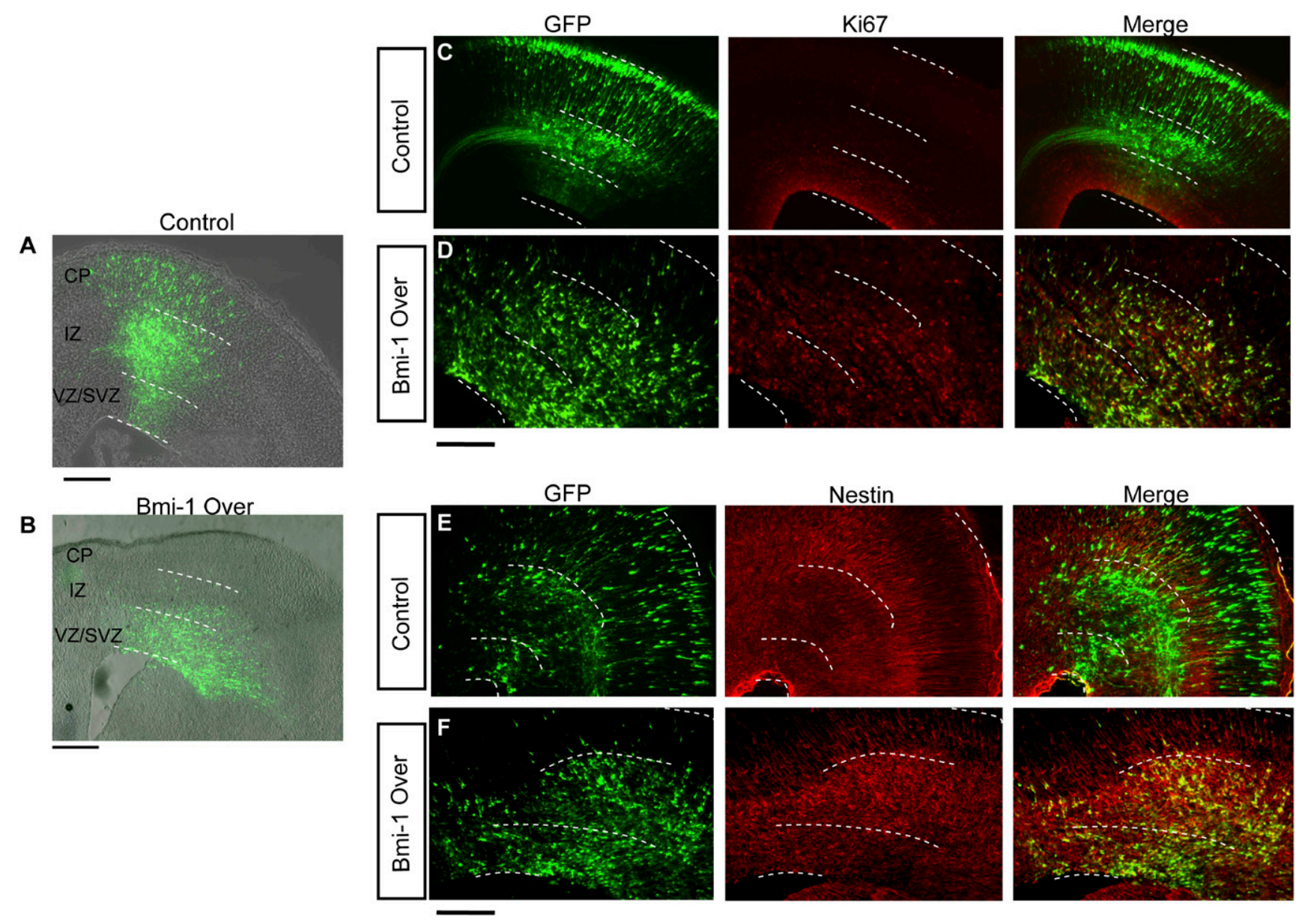

Figure 2. Bmi-1 overexpression stimulates embryonic cortical cell proliferation in vivo. In utero electroporation of Bmi-1 Over or EV lentiviral plasmids into E14 cortex and assessed at E17. $(A, B)$ Low-power images of control $(A)$ and Bmi-1-transduced $(B)$ cells in the E17 cerebral cortex showing the typical distribution of $\mathrm{GFP}^{+}$cells. White lines delineate regions of the developing cortex: (CP) cortical plate; (IZ) intermediate zone; (VZ/SVZ) ventricular and subventricular germinal zones; bar, $100 \mu \mathrm{m}$. (C) Control plasmid-expressing GFP ${ }^{+}$ cells are present in all layers from the VZ/SVZ to the CP, typical of normal development. Few control GFP ${ }^{+}$cells overlap with the proliferative marker Ki67 in the germinal VZ/SVZ region, consistent with most cells being post-mitotic. $(D) \mathrm{GFP}^{+} \mathrm{NPCs}$ transduced with Bmi-1 Over plasmid are numerous in the VZ/SVZ, while there are few cells in the CP. The majority of GFP ${ }^{+}$cells expressing Bmi-1

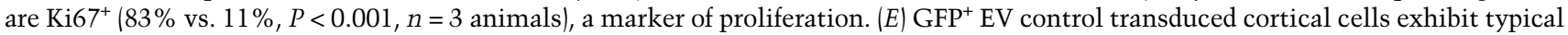
staining for the progenitor marker Nestin with radial fibers extending into the CP. Few Nestin ${ }^{+} \mathrm{GFP}^{+}$cells are present in the VZ/SVZ, while many $\mathrm{GFP}^{+}$cells are aligned with Nestin ${ }^{+}$fibers in the developing $\mathrm{CP}$, consistent with radial migration. $(F)$ After Bmi-1 Over transduction, the majority of $\mathrm{GFP}^{+}$cells remain below the $\mathrm{CP}$, most are Nestin ${ }^{+}(71 \%$ vs. $16 \%$ in control, $P<0.001, n=3$ animals), and the Nestin staining is disorganized. Bar, $50 \mu \mathrm{m}$.

numbers of both neurons and glia upon exposure to the same differentiation conditions (Fig. 4D-I). Many of the resulting SVZ cells stained for the immature neuronal marker $\beta$-tubulin III (55\%) (Fig. 4E,G) and for the more mature neuronal markers Map2 (49\%) (Fig. 4F) and NeuN $(22 \%)$ (Fig. 4H). To assess whether these neurons expressed molecules needed for synaptic transmission, we stained for synapsin and found that $14 \%$ of the Map2 $2^{+}$ cells contained synapsin, present at the tips of their processes (Fig. 4F, inset).

In the adult SVZ, NPCs normally produce neurons that migrate to the olfactory bulb, where they differentiate into GABAergic interneurons. Interestingly, after Bmi-1 overexpression, only a subpopulation $(56 \%)$ of the neu- rons generated were GABAergic, as assessed by GAD65/ 67 staining (Fig. 4I). Bmi-1 Over-expressing adult SVZ cells exhibited a variety of neuronal morphologies, including some with large cell bodies and long processes that were negative for GAD65/67 (Fig. 4I, arrows).

We then transduced adult SVZ cells in vivo with either Bmi-1 Over or control EV lentiviral vectors, by stereotaxically guided injections of lentiviral vectors. After $2 \mathrm{wk}$, cryostat sections of forebrain were examined. Bmi-1 overexpression stimulated adult SVZ cell proliferation compared with control, with dividing cells found in higher numbers in the SVZ and RMS (Fig. 4J [control], $M$ [Bmi-1 overexpression]) and an increase in GFPexpressing neurons in the olfactory bulb. The morphology 
$1^{\circ}$
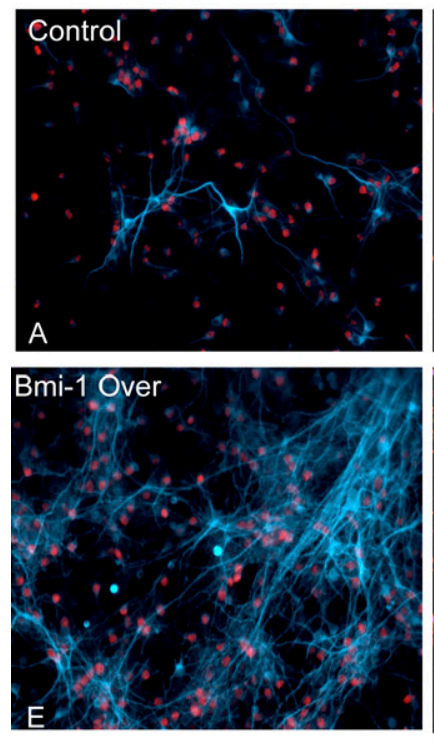

$2^{\circ}$
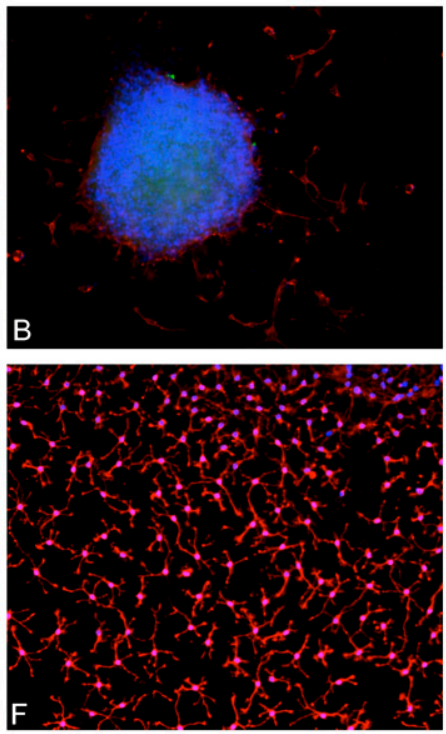

$3^{\circ}$
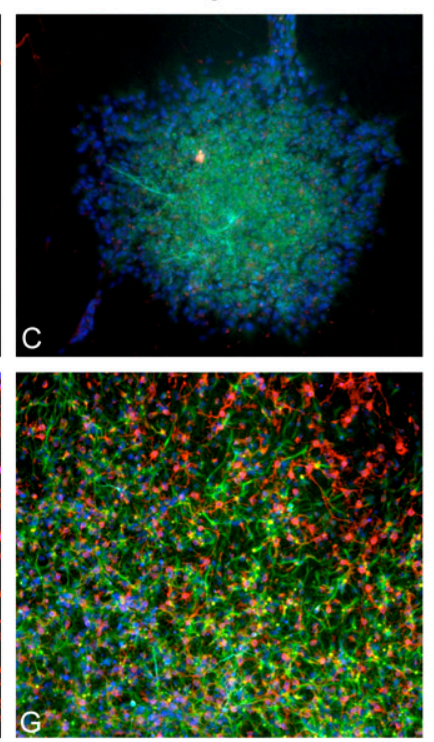

$4^{\circ}$
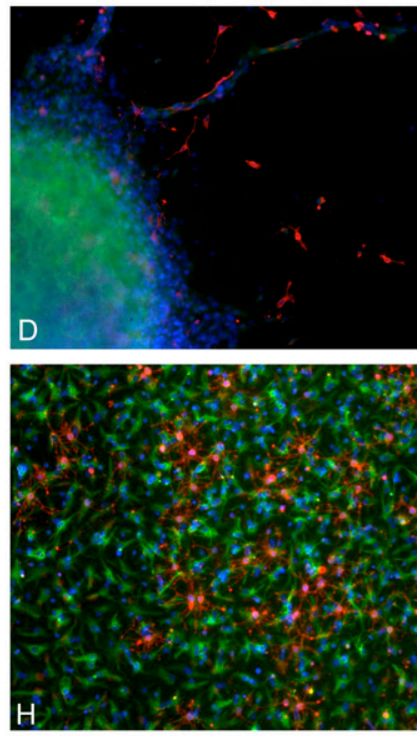

Figure 3. Bmi-1 overexpression increases expansion of embryonic cortical NPCs and maintains their normal cell type generation. $(A, E)$ Bmi-1 Over increased neuron generation in first passage spheres upon differentiation, and many were ER $81^{+}$, a cortical deep layer marker; (blue) $\beta$-tubulin III; (red) ER81. $(B-D)$ EV control. $(F-H)$ Bmi-1 Over. At later passages, both control and Bmi-1 Over-expressing spheres differentiated largely into glia, with enhanced numbers of oligodendrocyte lineage cells $(34 \%$ after Bmi-1 Over vs. $19 \%$ in EV control, $P<0.01, n=3$ experiments) and astrocytes (83\% after Bmi-1 Over vs. 59\% EV control, $P<0.01, n=3$ experiments) after Bmi-1 overexpression. $(B, F)$ (Blue) DAPI; (red) O4. $(C, D, G, H)$ (Blue) DAPI; (red) O4; (green) GFAP; (light blue) $\beta$-tubulin III. Bar, $30 \mu \mathrm{m}$.

of the neurons in the bulb after Bmi-1 overexpression appeared normal (Fig. 4K,L,N,O), and the distribution in the bulb was similar to EV-transduced cells.

\section{Bmi-1 increases Foxg1 expression in the NSC population}

Foxg1 is a forkhead transcription factor that is essential for forebrain development. Loss of Foxg1 severely reduces NPCs, and knockdown of Foxg1 in developing cortical cells inhibits cell proliferation (Q. Shen et al. 2006). These data indicate that Foxg1 could be important for NPC proliferation, and potentially NSC self-renewal. Moreover, Foxg1 can inhibit p21 levels to stimulate progenitor cell division, and as Bmi- 1 acts in part via suppression of p21 (Fasano et al. 2007), we examined the role of Foxg1 in Bmi-1-dependent NSC self-renewal.

We first examined whether Foxg1 is expressed in forebrain germinal zones throughout life. Its expression is well-established in developing telencephalic germinal zones (Hatini et al. 1994), but is less well described in the adult. Using a Foxg1 antibody (Irioka et al. 2005), we found that Foxg1 protein is expressed in a subpopulation of adult SVZ cells as well as many cells in the rostral migratory stream where progenitor cells and neuroblasts migrate toward the olfactory bulb (Supplemental Fig. $2 \mathrm{~A}, \mathrm{~B})$. NSCs in the SVZ express GFAP, but not all the $\mathrm{GFAP}^{+}$cells are stem cells (Doetsch et al. 1999). Some of the SVZ GFAP ${ }^{+}$cells were Foxg $1^{+}$(Supplemental Fig. 2C). We double-labeled adult SVZ sections for Bmi-1 and Foxg1 and found a similar staining pattern, with many
Foxg1/Bmi-1 double-positive cells (Supplemental Fig. 2D). Hence, Foxg1 protein is present in forebrain germinal zones throughout life and is present in a subpopulation of $\mathrm{GFAP}^{+}$cells, and frequently colocalizes with Bmi-1.

We next examined whether there was a functional relationship between Bmi-1 and Foxg1. After Bmi-1 overexpression in E11 and adult NPCs, we detected an increase in Foxg1 transcript levels at $7 \mathrm{~d}$ (Fig. 5A), and while we could not measure an increase in Foxg1 protein in E11 cells (possibly due to Foxg1 already being strongly expressed), there was a notable increase in Foxg1 transcript and protein in adult SVZ cells, along with a decrease in p21 expression, compared with EV control (Fig. 5A,B; Supplemental Fig. 3A). Conversely, when E11 cortical cells were transduced with a verified Foxg1 shRNA construct (Q. Shen et al. 2006), Bmi-1 expression levels were reduced at $7 \mathrm{~d}$ (Fig. 5B), suggesting a possible regulatory feedback loop between these two factors, which will be a worthwhile point for further investigation.

To test whether the increase in Foxg1 expression occurs rapidly after the stimulation of Bmi- 1 expression, embryonic cortical cells were transduced with the Bmi- 1 overexpression construct, and Foxg1 levels were measured at $48 \mathrm{~h}$, which is soon after the construct is expressed. At this time point, we found that there was no increase in cell number compared with control (data not shown), consistent with prior observations (Fasano et al. 2007), and so it is unlikely that any measured change in Foxg1 expression is due to changes in composition of the cell population, such as an increase in the 
Figure 4. Bmi-1 maintains multipotency of adult SVZ NSCs. $(A-C)$ In control conditions, fifthpassage NPCs are numerous and healthy (phase picture, $A$ ) but they lose their multipotency, generating few if any $\beta$-tubulin $\mathrm{III}^{+}$neurons, $(B$, blue $)$ and few oligodendrocytes, revealed by $\mathrm{O} 4$ staining $(C$, red). $(B$, inset $)$ Nestin staining in control neurosphere cells demonstrates their progenitor state (green, Nestin). (D) Adult SVZ NPCs expressing Bmi-1 remain multipotent and generate clones containing progeny of all three major CNS cell types ([red] O4; [blue] GFAP; [green] $\beta$-tubulin III), including numerous neurons staining with the following markers: $(E) \beta$-tubulin III $(55 \%$ vs. $9 \%$ control, $P<0.001, n=4)$ ). $(F)$ Map-2 (49\% vs. $5 \%$ control $P<0.001, n=4)$. ( $F$, inset $)$ Some neurons stained with Synapsin1, a protein expressed on synaptic vesicles, localized at the end of neuronal branches ([green] Map-2; [red] synapsin1, 14\% vs. $2 \%$ control, $P<0.001, n=3)$. $(G)$ High-power $\beta$ tubulin III staining showing neuronal morphologies; (red) $\beta$-tubulin III; (green) GFP. $(H)$ Neurons stained with NeuN $(22 \%$ vs. $4 \%$ control, $P<$ 0.001 ); (red) NeuN; (green) Map2; (blue) DAPI. $(I)$ Fifty-six percent of the neurons generated are GABAergic ( $\beta$-tubulin, blue III; GAD65/67, red), and the remainder were negative for GAD65/67 (arrows). (J-O) Bmi-1 Over in the adult SVZ led to more proliferating $\left(\mathrm{Ki}^{+} \mathrm{7}^{+}\right)$cells in the SVZ and RMS $(M)$ as well as significantly more in the olfactory bulb $(N, O)$ compared with EV control virus injections $\left(J-L, 37.5\right.$ vs. $9.9 \mathrm{GFP}^{+}$cells per field, $P<0.05, n=4$ animals); (red) Ki67. $(L, O)$ High-power images of transduced, $\mathrm{GFP}^{+}$cells in the olfactory bulb. Bars: $A-C, F$ (inset), $G-I, L, O, 20$ $\mu \mathrm{m} ; D-F, J, K, M, N, 50 \mu \mathrm{m}_{;}$, , $B$, inset, $100 \mu \mathrm{m}$.
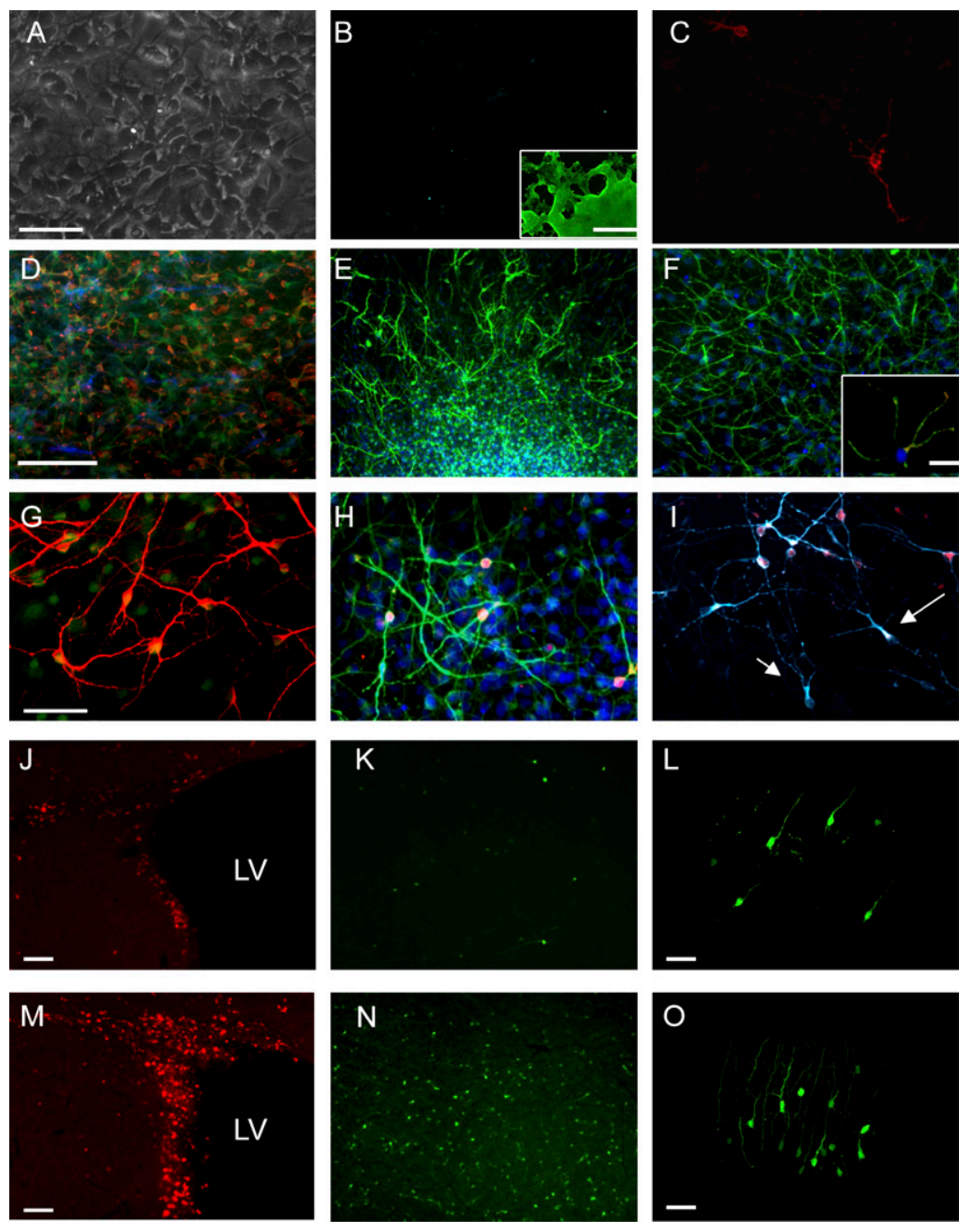

number of progenitor cells. We found that Foxg1 transcript levels increased by $57 \%$ at $48 \mathrm{~h}$ (Fig. $5 \mathrm{C}$ ), suggesting that this is an early response to Bmi-1 overexpression.

Embryonic forebrain cultures include self-renewing NSCs, restricted progenitor cells, and differentiated progeny. We examined whether sustained Bmi-1 expression alters the proportions of these cell types. We isolated embryonic cortical cells and transduced them with Bmi-1 or EV control virus. After 1 wk in culture, the cells were dissociated, labeled with LeX-a surface marker that labels NSCs and the more proliferative restricted progenitors but not differentiated cells /Capela and Temple 2006) - and separated them by FACS. Bmi-1 overexpression caused a $39 \%$ increase in $\mathrm{LeX}^{+}$cells and a $35 \%$ decrease in LeX ${ }^{-}$cells, showing that Bmi-1 expands the NPC population and reduces cell differentiation (Fig. 5D). Also as expected, overexpression of Bmi-1 resulted in an increase in Foxgl expression in the total cell population. However, when analyzed separately, the LeX ${ }^{+}$cells overexpressing Bmi-1 had significantly increased Foxgl expression compared with EV control, while the LeX-ve cells overexpressing Bmi-1 had a significantly lower level of Foxg1 expression compared with the EV control (Fig. 5E).
Thus, forced expression of Bmi-1 maintains the NPC population and stimulates an increase in Foxg1 levels specifically in these proliferating progenitor cells.

\section{Foxg1 is necessary for Bmi-1-stimulated forebrain NSC self-renewal}

To find out if the measured increase in Foxg1 was important for NSC self-renewal, we enforced expression of Bmi-1 in E11, E16 cortical and adult SVZ NPCs and concomitantly treated the cells with verified Foxg1 shRNA vectors (Q. Shen et al. 2006). This markedly reduced primary neurosphere frequency compared with control, and the effect increased with stage (Fig. 6A), as observed after Bmi-1 shRNA treatment of NSCs (Fasano et al. 2007). To further confirm this, neurospheres that were already overexpressing Bmi-1 and actively growing were subcloned and then transduced with EV control or Foxg1 shRNA. Secondary neurosphere formation was again significantly inhibited after Foxgl shRNA treatment, attesting to its critical role in NSC self-renewal (Fig. 6B).

To explore this interaction in vivo, we electroporated either Foxg1 shRNA plasmid alone or Bmi-1 overexpression 
A

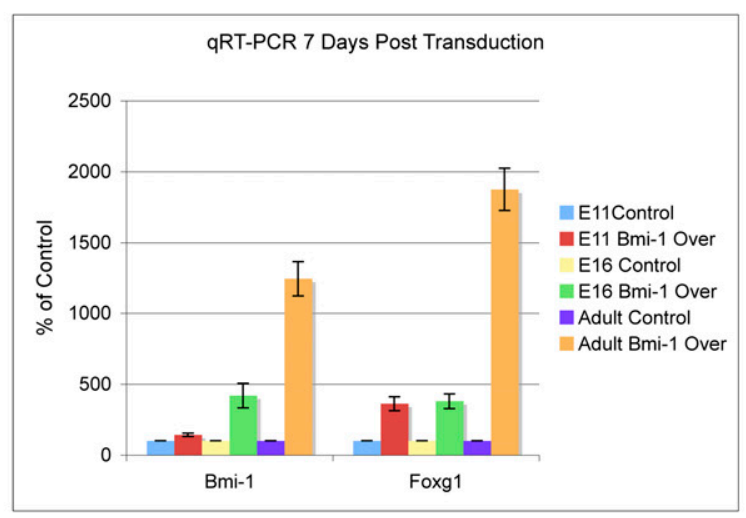

B

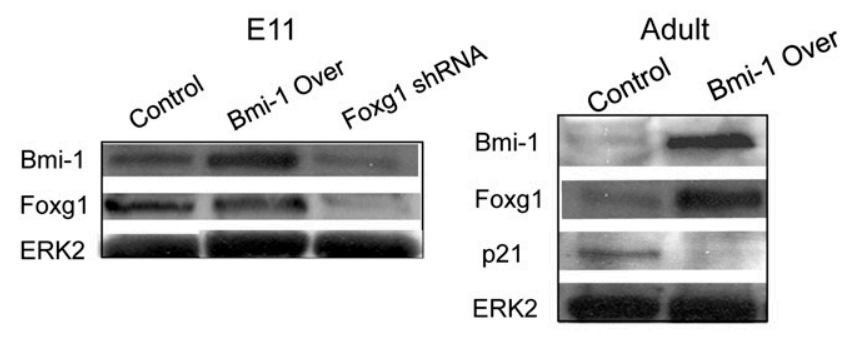

C

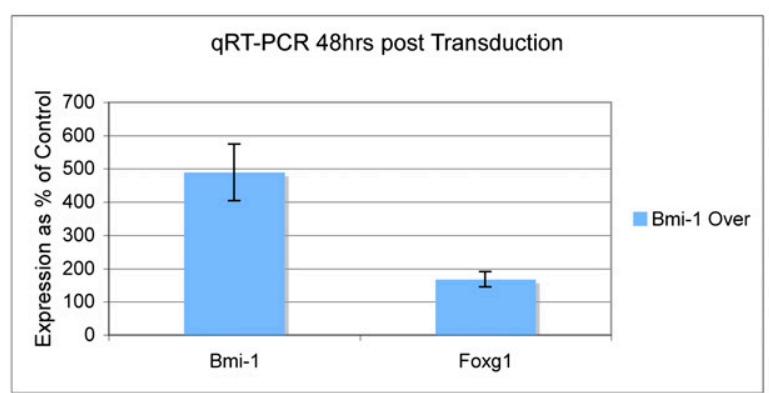

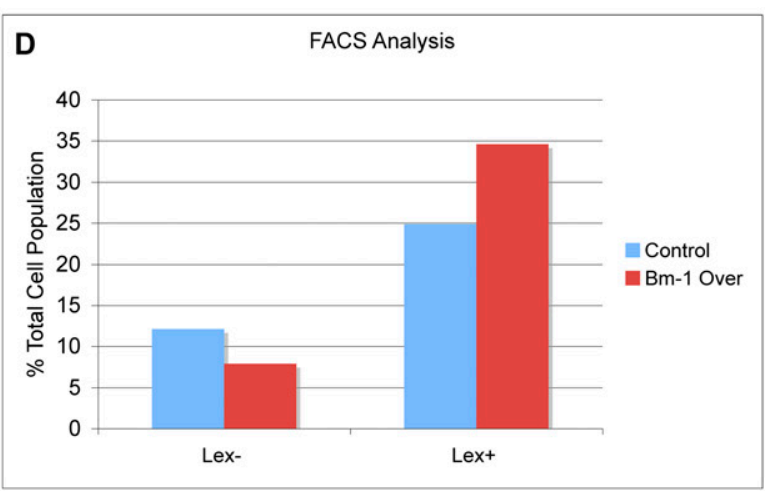

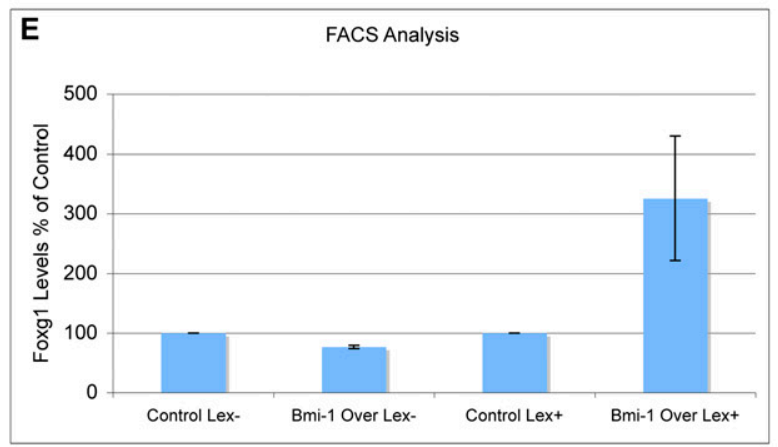

Figure 5. Bmi-1 overexpression increases Foxg1 levels in the NSC population. $(A)$ NPCs from embryonic cerebral cortex and adult SVZ transduced with lentiviral EV control or Bmi-1 Over vectors were grown in adherent conditions for $7 \mathrm{~d}$. Transcript levels of Bmi-1 and Foxg1 were measured ( $n=3$ experiments). Values are expressed as percent of control. (B) Following the same protocol in $A$, after $7 \mathrm{~d}$, protein was isolated and measured by Western blot $(n=3) .(C)$ E16 NPCs were transduced with Bmi-1, and soon after the vector was transcribed, Foxg1 levels increase $(n=3$ experiments). Values are expressed as percent of control. $(D)$ FACS analysis shows that after Bmi-1 overexpression the $\mathrm{LeX}^{+}$population increases, consistent with a greater proportion of progenitor cells. (E) E14 cortical cells were transduced with Bmi-1 Over or EV control lentiviruses, and then LeX $^{+}$and LeX cells were separated by FACS. After Bmi-1 Over treatment, the LeX-population showed a decrease in the levels of Foxg1, while in the LeX ${ }^{+}$population, there was an increase $(n=2$ for FACS and all qRT-PCRs were performed in triplicate). Values are expressed as percent of control.

and Foxg1 shRNA plasmids together into mouse embryonic cortex at E14 (Fig. 6C-E). Electroporation of Foxg1 shRNA alone resulted in few cells in the VZ, with most $\mathrm{GFP}^{+}$cells in the intermediate zone (IZ) and the CP, indicating that the transduced NPCs had largely exited the germinal zones (Fig. 6C; Supplemental Fig. 1B). This is consistent with the Foxg1 knockout phenotype and is similar to the action of Bmi-1 shRNA in vivo (Xuan et al. 1995; Fasano et al. 2007). Cotransfection of Bmi-1 and Foxg1 shRNA plasmids prevented the dramatic expansion of progenitor cells seen with Bmi-1 alone, producing a phenotype similar to Foxg1 shRNA alone (Fig. 6D,E; Supplemental Fig. 1A,B).

We confirmed these observations using NSCs derived from mouse embryonic stem (ES) cells lacking one or both copies of Foxg1. Prior studies have shown that Foxg1 heterozygotes have impaired neural progenitor proliferation (L. Shen et al. 2006). After one passage, Bmi-1 overexpression increased neurosphere frequency in the $+/+$ control cells. However, in either the $+/-$ or $-/-$ ESderived NSCs, Bmi-1 overexpression was unable to compensate for the proliferative defects seen in the mutant cells (Supplemental Fig. 3). We conclude that Bmi-1dependent self-renewal requires a normal level of Foxg1 function.

Both Bmi-1 and Foxg1 have been shown to regulate p21 in NPCs (Seoane et al. 2004; Fasano et al. 2007). Given this, we investigated the functional contribution of $\mathrm{p} 21$ to the Bmi-1 and Foxg1 relationship in NSC self-renewal. E14.5 NPCs were isolated, transduced with EV control or p21 shRNA constructs, and plated in neurosphere-forming conditions. After $7 \mathrm{~d}$, the neurospheres were dissociated, 

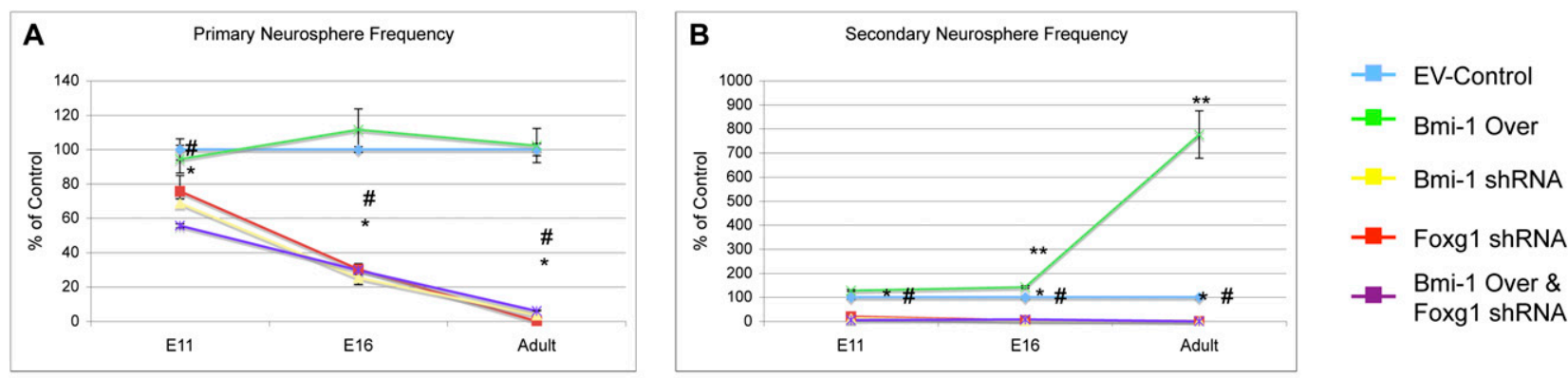

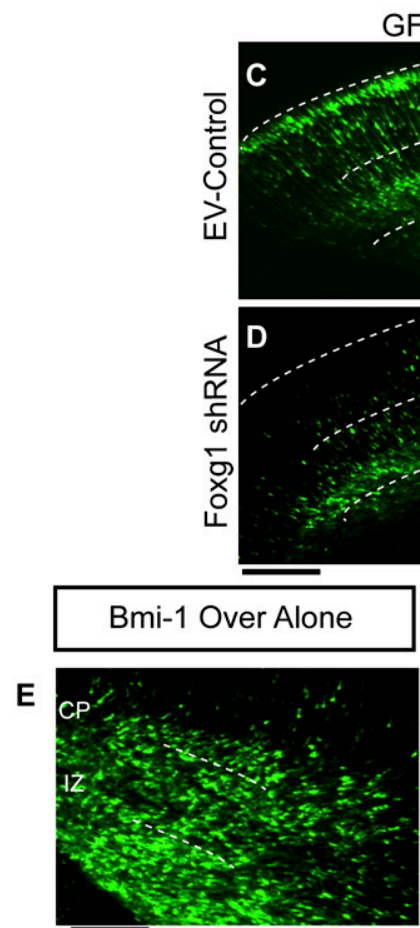

Bmi-1 Over
GFP

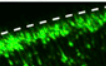

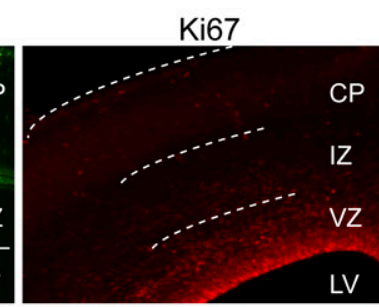
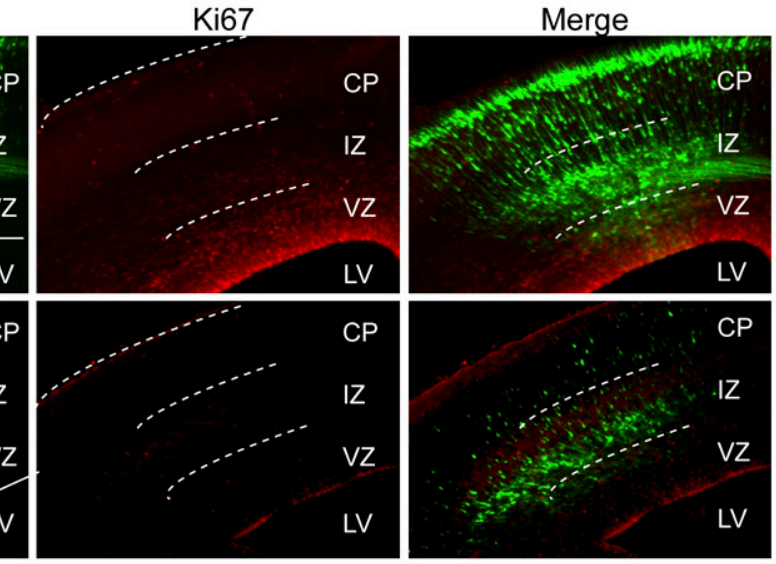

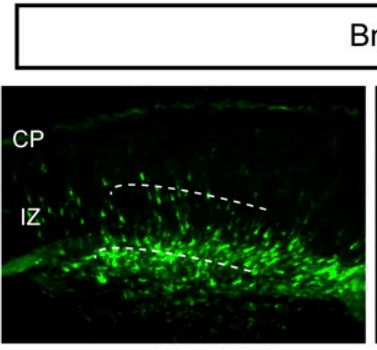

Foxg1 shRNA

Bmi-1 Over \& Foxg1 shRNA

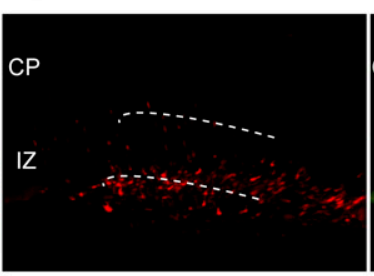

Bmi-1 Over

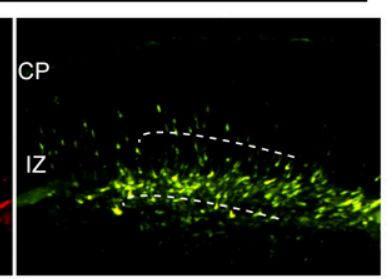

Merge

Figure 6. Bmi-1-mediated expansion is dependent on Foxg1 in vitro and vivo. $(A, B)$ Neurospheres isolated from embryonic cortex and adult SVZ were transduced with Bmi-1 Over and Foxg1 shRNA plasmids, singly or together. Foxg1 shRNA treatment decreased neurosphere frequency and abolished the increased NPC expansion seen with Bmi-1 Over $(A$, primary spheres: significance vs. control, $\left[{ }^{\star}\right] P<0.01$ or vs. Bmi- 1 expression alone, $[\#] p<0.005 ; B$, secondary spheres: $\left[{ }^{\star}\right] p<0.001$ significance vs. control, [\#] $P<0.001$ vs. Bmi- 1 Over, $\left.{ }^{\star \star}\right] P<0.01$ vs. control). The phenotype was more severe for adult SVZ cells treated with Foxg1shRNA than other stages. Values are expressed as percent of control. Bar, $100 \mu \mathrm{m} .(C, D)$ Electroporation of Foxg1 shRNA in vivo reduces proliferating NPCs: GFP ${ }^{+}$cells are largely absent from the VZ/SVZ, and most are Ki67-negative. Bar, $50 \mu \mathrm{m}$. $(E)$ As seen in Figure 2, electroporation of Bmi-1 Over plasmids in vivo expands NPCs in the VZ/SVZ. When combined with Foxg1 shRNA in vivo, this expansion is abolished, demonstrating that Foxg1 is required for Bmi-1-mediated expansion in vivo ([GFP] Foxg1 shRNA; [dsRed] Bmi-1 Over vector; [yellow] merge; all cells examined were found to have received both Foxg1shRNA and Bmi-1 Over plasmids). Bar, $50 \mu \mathrm{m}$.

and the resulting single cells were transduced with EV control, Bmi-1 Over, Foxg1 shRNA, or Bmi-1 Over together with Foxg1 shRNA constructs. After $1 \mathrm{wk}$, the neurospheres were counted. As expected, after Foxg1 shRNA alone or Bmi-1 Over and Foxg1 shRNA together, significantly fewer neurospheres were generated. However, this phenotype was significantly rescued when these constructs were added to NPCs that had been pretreated with p21 shRNA (Supplemental Fig. 4). These data demonstrate that Bmi-1 and Foxg1 cooperate to regulate NPC self-renewal and that an important aspect of their interaction is focused on the regulation of $\mathrm{p} 21$.

\section{Foxg1 overexpression stimulates NPC survival}

Bmi-1-stimulated self-renewal is dependent on Foxg1, and it is possible that they both stimulate self-renewal but operate in independent pathways. To address this possibility, we enforced expression of Foxg1 in NPCs and assessed self-renewal using the neurosphere assay. Overexpression of Foxg1 in SVZ cells increased secondary neurosphere frequency (35\% above control), but not as much as overexpression of Bmi-1 (77\% above control) (Fig. 7A). The neurosphere assay can expand both stem cell and non-stem cell populations at early passages, and 

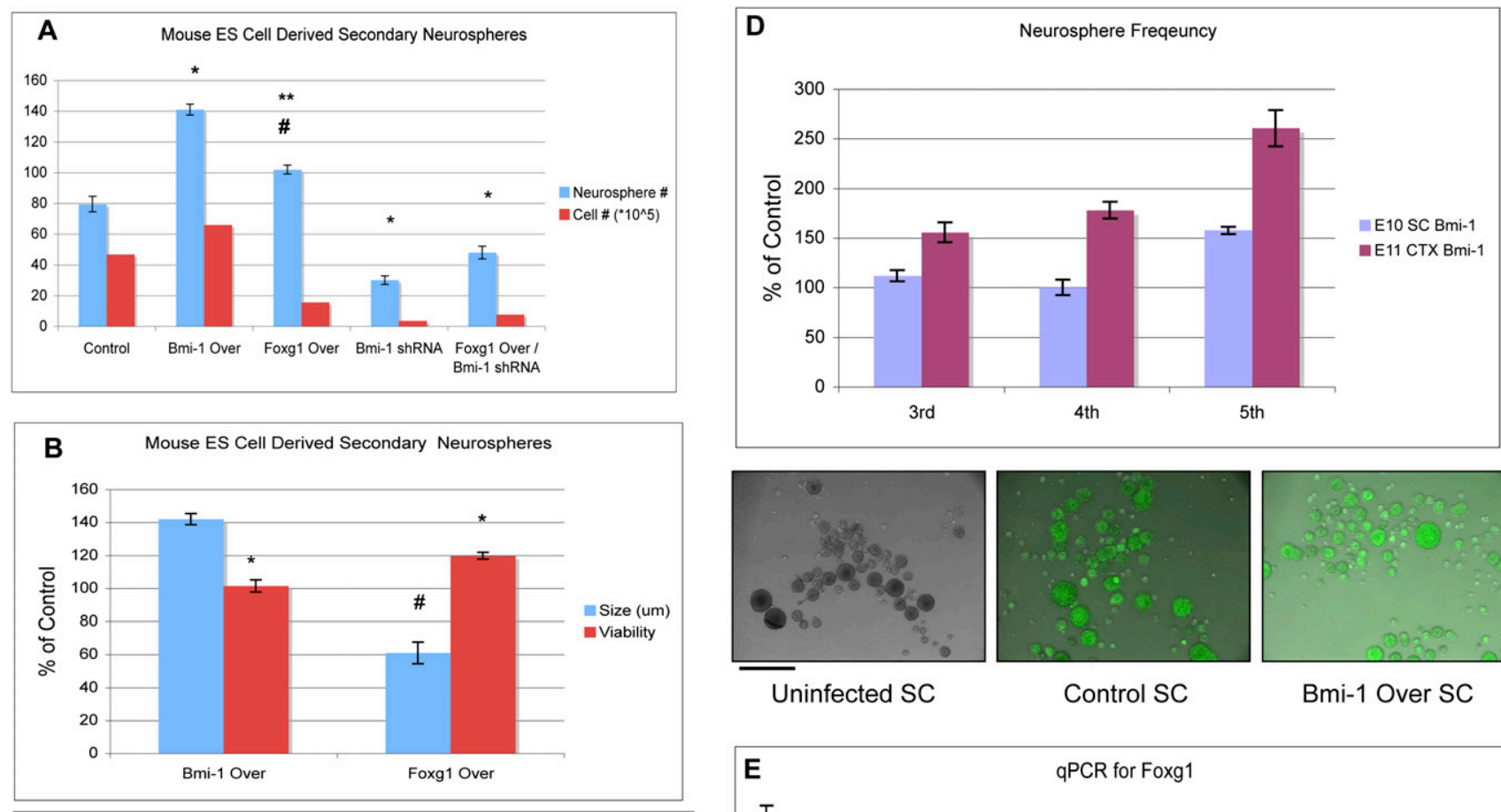

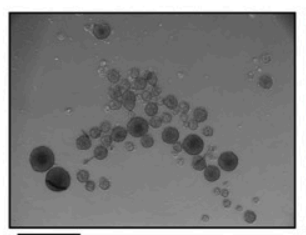

Uninfected SC

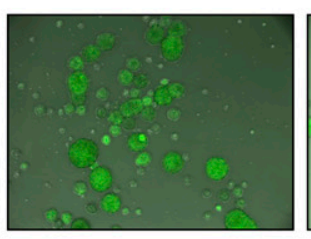

Control SC

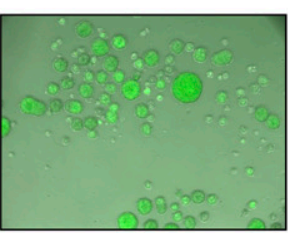

Bmi-1 Over SC

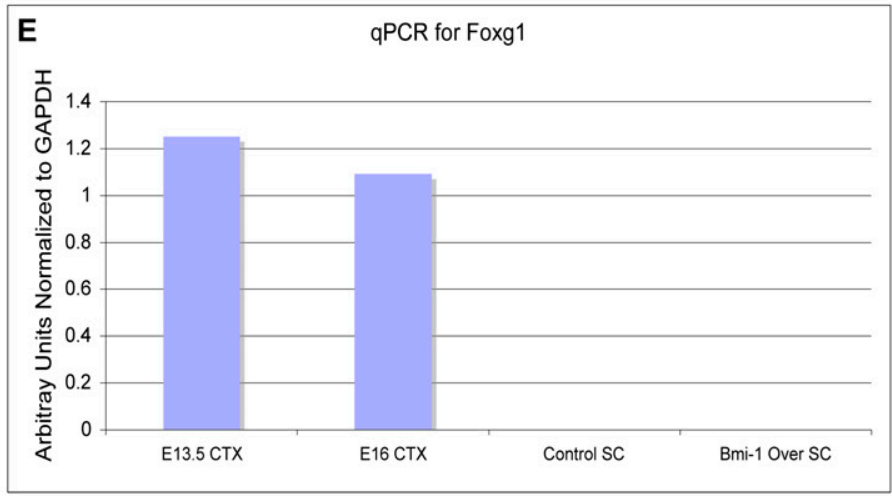

Figure 7. Foxg1 is important for robust forebrain but not spinal cord NSC growth after Bmi-1 overexpression. $(A)$ NPCs were derived from mouse ES cells and then propagated as neurospheres. After $1 \mathrm{wk}$ in culture, the neurospheres were subcloned, and the number of secondary neurospheres was counted at day 7 , as a measure of self-renewal. Bmi-1 overexpression significantly increased neurosphere number above control $\left.\left(n=3,{ }^{\star}\right] P<0.01\right)$ and increased total cell number. Foxg1 overexpression also increased NS number $(n=3$, [\#] $P<$ 0.05) but significantly less compared with Bmi-1 Over $\left(\left[{ }^{\star \star}\right] P<0.05\right)$. Foxg1-overexpressing spheres contained fewer cells than those from EV control and Bmi-1 Over conditions. Bmi-1 shRNA treatment decreased both neurosphere number $\left(n=3\right.$, $\left.\left.{ }^{\star}\right] P<0.01\right)$ and cell number, a deficit not rescued by Foxg1 overexpression. $(B)$ Neurosphere size and cell viability as percent of control. Neurospheres overexpressing Bmi-1 were larger, and the cells had similar viability compared with control $\left(n=3,\left[^{\star}\right] P<0.05\right)$, as measured by trypan blue exclusion. In contrast, neurospheres overexpressing Foxgl were smaller and had increased cell viability compared with control $(n=$ $\left.\left.3,{ }^{\star}\right] P<0.05,[\#] P<0.01\right)$. Values are expressed as percent of control. $(C)$ The same experiment as in $A$ was performed in primary adult SVZ neurosphere cultures with similar results $\left(n=3,{ }^{\star}\right] P<0.01,[\#] P<0.05$ vs. EV control; $\left.\left.{ }^{\star}{ }^{\star}\right]\right] P<0.05$ vs. Bmi- 1 Over $)$. Bar, $100 \mu \mathrm{m}$. $(D)$ Neurospheres from spinal cord (SC) were passaged five times. Neurosphere frequency increased less dramatically compared with embryonic cortex after Bmi-1 Over $\left(n=3,\left[^{\star}\right] P<0.01\right.$ significance vs. control; [\#] $P<0.01$ vs. Bmi-1 Over). Values are expressed as percent of control. (E) qRT-PCR shows lack of Foxg1 expression in stem cell neurospheres.

it has been suggested that assessing growth up to five passages is a better assessment of self-renewal (Reynolds and Rietze 2005|.With further passaging, neurosphere frequency after Foxg1 overexpression did not continue to increase (data not shown), whereas it did after Bmi-1 overexpression (Fig. 1). Moreover, Foxg1-overexpressing spheres were smaller in diameter and composed of fewer cells (Fig. 7B). Prior studies have shown that Foxg1 expression in vivo in chick brain stimulates NPC survival but not proliferation (Ahlgren et al. 2003). The smaller primary neurospheres observed after Foxg1 overexpression are consistent with an increase in survival of progenitor cells that would normally die off in the neurosphere assay, as the non-adherent conditions are not conducive for their survival (Reynolds and Weiss 1992). In addition, we observed an increase in cell viability by trypan blue exclusion after Foxg1 overexpression compared with either the control or Bmi-1 overexpression conditions (Fig. 
7B,C). These experiments demonstrate that Foxg1 does not itself notably stimulate NSC self-renewal, but indicate that it is important for forebrain NPC survival, making Foxg1 an important effector of Bmi-1 stimulated self-renewal.

\section{Bmi-1 stimulation of self-renewal is region-specific}

To determine whether Bmi-1 can regulate NPC selfrenewal from other CNS regions, we isolated NPCs from embryonic spinal cord, a region of the nervous system that has more limited expansion during development and lacks active adult neurogenesis, and transduced them with EV control and Bmi-1 Over vectors. Following the same procedure described in Figure 1, control and Bmi-1overexpressing neurospheres were passaged five times, and the frequency of neurosphere formation was assessed at each passage. There was an increase in spinal cord neurosphere frequency after Bmi-1 overexpression, but it was delayed and much less robust than that observed using forebrain NPCs (Fig. 7D). Spinal cord cells do not normally express Foxg1 (Fig. 7E; Xuan et al. 1995). We found that Foxgl expression was not induced in spinal cord progenitor cells after Bmi-1 overexpression, and as expected, Foxg1 shRNA had no effect on spinal cord neurosphere formation, further validating the specificity of the action of Foxg1 shRNA on forebrain NPC selfrenewal (data not shown). Moreover, we found that enforcing both Bmi-1 and Foxgl overexpression in spinal cord progenitor cells did not enhance their ability to self-renew above Bmi-1 Over alone (data not shown), demonstrating that Foxg1 is not sufficient to confer a forebrain-like selfrenewing program in non-forebrain NPCs. In conclusion, Bmi-1 overexpression stimulates growth of spinal cord NPCs, but less effectively than those from the forebrain, indicating that Bmi-1 interacts with regional programs to regulate appropriate levels of NSC self-renewal.

\section{Discussion}

The mammalian forebrain expands more than other CNS regions during development, and it maintains a population of neurogenic stem cells throughout life. Perhaps these phenomena are related and can be traced to the forebrain having a more robust mechanism for progenitor self-renewal than other regions. Previous knockout and knockdown studies identified Bmi-1 as a critical mediator of NSC self-renewal. Here we demonstrate that Bmi-1 overexpression increases self-renewal of forebrain NSCs significantly more than spinal cord NSCs. Moreover, Bmi-1 overexpression in forebrain cells rapidly up-regulates the forebrain-specific transcription factor Foxg1, and we show that Foxg1 is necessary for forebrain NSC selfrenewal. These data provide insight into how regional NSCs are maintained. In addition, we demonstrate that Bmi-1 overexpression is a useful method for generating abundant forebrain neurons ex vivo.

It is somewhat surprising that despite the robust cell division of forebrain NSCs elicited by enforced Bmi-1 expression, the cells are not prevented from differentiat- ing. Rather, at each age tested, Bmi-1-overexpressing neurospheres differentiated readily in vitro to produce the major classes of cells that they would normally produce in vivo. We found that after in utero electroporation of Bmi-1 Over plasmid into cortical NPCs in vivo, their proliferation was enhanced but differentiation was inhibited, over a 3-d period. When we allowed these animals to develop for longer periods, into the first week after birth, large growths were observed in some brains (data not shown), indicating that prolonged expression of Bmi-1 could result in tumor formation. In the future, inspection of these growths should indicate the types of differentiated cells that are included. In the adult SVZ, overexpression of Bmi-1 produces abnormally high levels of cell proliferation throughout the RMS (Fig. 4M-O); perhaps with longer exposure, more significant growth might occur. Nevertheless, Bmi-1-overexpressing cells in the adult could differentiate into olfactory bulb cells that were distributed in the correct layers and had apparently normal morphology. Together, these results indicate that overexpression of Bmi-1 expands the proliferative compartment, but is not inhibitory to normal differentiation signals. These observations are interesting in the light of the possible role of Bmi-1 in tumor formation. High levels of Bmi-1 are critical for the tumorigenicity of neuroblastoma cells (Cui et al. 2007), yet in some cases brain tumor-propagating cells readily respond to normal stem cell differentiation signals such as bone morpogenic proteins (Piccirillo et al. 2006). It remains to be determined whether overexpression of Bmi-1 can make a normal NPC become tumorigenic, and whether its interaction with regional regulators such as Foxg1 provide suitable therapeutic targets for combating self-renewal of tumorpropagating cells. Hence, the outcome of these in vivo overexpression experiments will be interesting to explore further.

Adult SVZ cells normally make neurons throughout life as well as glia in vivo, but when removed from the neurogenic niche and plated in tissue culture, their ability to make neurons declines rapidly. In contrast, when Bmi-1 levels are increased by lentiviral transduction, adult SVZ cells retain their ability to make neurons even outside of the niche, and they also generate astrocytes and oligodendrocytes. We speculate that environmental niche factors that promote NSC self-renewal and maintain their neurogenicity likely operate, at least in part, by maintaining a critical level of Bmi-1 expression. Thus, artificially enhancing Bmi-1 expression can maintain self-renewal and multipotency ex vivo.

We noted that upon differentiation, Bmi-1-overexpressing adult SVZ cells produced a variety of neuron morphologies, which could point to enhanced developmental potency. There are indications that adult SVZ cells are not just restricted to making olfactory neurons: They produce striatal neurons after addition of BDNF and Noggin (Chmielnicki et al. 2004), or cells that migrate toward areas of ischemic damage in the parenchyma (Zhang et al. 2007). Hence, Bmi-1 overexpression might reveal a broader repertoire of neurogenesis. The fact that enforced Bmi-1 expression allows expanded adult SVZ 
cells to generate numerous neurons-as many as $50 \%$ after $1 \mathrm{mo}$ in culture and five passages-makes this a highly useful technique to generate forebrain neurons ex vivo, for example, to model diseases that affect these cells.

The increase in Foxg1 levels and in NSC self-renewal after Bmi-1 overexpression was more pronounced with stage, pointing to an age-related effect. Alternatively, it could reflect dosage, if Bmi-1 transcripts are induced to much higher levels in adult cells compared with embryonic cells. However, we found that there was only a 1.3fold increase in the Bmi-1 transcript level in adult cells compared with E16 cortical cells after Bmi-1 Over treatment. Consequently, we conclude that Bmi-1 overexpression has a more dramatic effect in the adult due to agerelated differences in the responding cells, consistent with the greater impact of Bmi-1 knockdown in adult compared with embryonic cells (Fasano et al. 2007). It is understandable that proliferation controls vary with stage, as embryonic cells must divide extensively to build the nervous system, while adult SVZ progenitors may divide as little as once per week to maintain the mature olfactory system, and are tightly constrained from overgrowth that could result in tumor formation.

The central role of Foxg1 in telencephalic development, its function in regulating cell cycling via p21 repression, and the fact that we, and others, observe its expression in germinal zones throughout life make it an ideal candidate for a stem cell regulator throughout life. Indeed, knocking down Foxg1 in embryonic cortical cells causes a depletion of the stem/progenitor pool and induces neural differentiation (Q. Shen et al. 2006). Furthermore, Foxg1 is expressed in adult germinal zones, and we show that Foxg1 protein is present in a subpopulation of $\mathrm{GFAP}^{+}$cells in the adult SVZ. Given that not all $\mathrm{GFAP}^{+}$cells are SVZ stem cells, it is possible that the Foxg $1^{+}$subpopulation are stem cells or actively dividing progenitor cells, which should be a point of future studies.

We found that Bmi-1 positively regulates self-renewal and is accompanied by Foxg1 up-regulation, by a mechanism that remains to be determined. Without Foxg1, NPCs deplete, and Bmi-1 expression cannot override the deficit, suggesting that Bmil and Foxgl cooperate to regulate forebrain NSC maintenance. This phenotype can be partially rescued by knocking down p21, demonstrating its central role in NSC self-renewal, as a focal point of both Bmi-1 and Foxg1 action. Bmi-1 is expressed in the developing spinal cord (Fig. 7D); however, when we overexpressed Bmi-1 in spinal cord NPCs, we did not see the dramatic expansion observed with forebrain NPCs after a similar treatment. We conclude that Bmi-1 does not simply increase the proliferation of all progenitor cells, but rather operates in a cell-type-specific context to achieve appropriate levels of NSC expansion. Prolonged expression of Bmi-1 and Foxg1 into adulthood ensures continued Bmi-1-driven expansion of forebrain NSCs throughout life, and positive regulation between these two proteins could help maintain the progenitor population in the forebrain over the long term. Bmi-1 stimulates growth of other CNS regions during develop- ment, such as cerebellum and retina (Leung et al. 2004; Zencak et al. 2006), and it seems likely that it will interface with regional growth-regulating genes in those locales to orchestrate appropriate regional development. Understanding region-specific NSC maintenance will be extremely important for generating specific cell types for therapeutics and should provide insight into tumor generation from different CNS regions.

\section{Materials and methods}

\section{Primary NPC culture}

NPCs were isolated from the embryonic cortex, spinal cord, and adult SVZ as described previously (Qian et al. 2000; Sun et al. 2005; Lowry et al. 2008). Cells were grown in non-adherent conditions in the presence of $20 \mathrm{ng} / \mathrm{mL}$ FGF2 and $20 \mathrm{ng} / \mathrm{mL}$ EGF for $7 \mathrm{~d}$ to form neurospheres (Reynolds and Weiss 1992). To differentiate NPCs, neurospheres were plated onto poly-l-lysinecoated tissue culture plates, growth factors were removed, and cell type generation was assessed $14 \mathrm{~d}$ after plating.

\section{Neurosphere culture}

Single dissociated cortical cells were cultured in uncoated 6-well plates for $7 \mathrm{~d}$ in serum-free medium containing DMEM, N2, B27, $\mathrm{NAC}$, and $20 \mathrm{ng} / \mathrm{mL}$ each FGF2 and EGF. For subcloning, neurospheres were collected and gently dissociated using papain (Worthington) for $20 \mathrm{~min}$ at $37^{\circ} \mathrm{C}$ with gentle agitation. Cells were replated at the same cell density for each condition, and numbers of neuropheres were counted after $7 \mathrm{~d}$ by microscopy.

\section{Neurosphere differentiation}

Neurospheres were removed from cell culture and plated into poly-l-lysine-coated six-well plates (Costar) in serum-free culture medium (described above) but without EGF or FGF2. Neurospheres were allowed to differentiate for $14 \mathrm{~d}$ before being fixed for immunocytochemistry.

\section{ES cell-derived NPC cultures}

ESCs were neurally induced using a modified serum-free embryoid bodies (SFEBs) protocol (Watanabe et al. 2005). Briefly, SFEBs were generated by plating single-cell suspension of ESCs (50 K/ $\mathrm{mL}$ ) on ultra-low adherence dishes (Costar) in a 1:1 ratio of KSR:N2 media. (KSR and N2 media preparation is described in Perrier et al. [2004].) Medium was changed every other day. At day 5 , SFEBs were collected, dissociated into single cells after $1 \mathrm{~h}$ of incubation in Hanks Balanced Salt Solution, and plated on Polyornithine/Laminin-coated dishes at a density of 250,000 cells per square centimeter, in N2 medium supplemented with Sonic Hedgehog and FGF8 (200 ng/mL each). For NPC derivation, neuroectodermal cells were dissociated after an additional 2-3 $\mathrm{d}$ and replated in N2 medium supplemented with FGF2 and EGF $(20 \mathrm{ng} / \mathrm{mL})$. For neurosphere assays, cells were plated onto ultralow adherence dishes at either 2000, 5000 or 10,000 cells per milliliter in DMEM supplemented with N2 supplement (GIBCO $100 \times)$, B27 (GIBCO 100×), FGF2 (20 ng/mL), and EGF (20 ng/ $\mathrm{mL})$.

\section{Vector production and viral packaging}

A third-generation lentiviral vector (Lois et al. 2002) was modified to express a shRNA from the $\mathrm{H} 1$ promoter as described 
(Ivanova et al. 2006; Fasano et al. 2007). Foxg1 shRNA constructs were used as previously described (Q. Shen et al. 2006). The shRNA-expressing lentiviral plasmid was cotransfected with plasmids pVSV-G and pCMVd8.9 into 293FT cells. Viral-containing media were collected, filtered, and concentrated by ultracentrifugation. Viral titers were measured by serial dilution on NIH 3T3 cells followed by flow cytometric analysis after $72 \mathrm{~h}$. For viral transduction, lentiviral vectors at an MOI (multiplicity of infection) of 10 were added to dissociated cortical cells prior to or just after plating.

\section{Lentiviral overexpression}

The original lentiviral construct was derived from FUGW (Lois et al. 2002). The Bmi-1 and Foxg1 clones were obtained from OpenBiosystems and cloned into the modified vector driving transgene expression with a Ubquitin-C promoter and an IRES eGFP for visualization in cells. Viral transduction was accomplished as described above.

\section{Immunocytochemistry}

Cell cultures were fixed with $4 \%$ paraformaldehyde and immunostained overnight at $4{ }^{\circ} \mathrm{C}$ with primary antibody: $\beta$-tubulin III (1:800; Sigma), O4 (1:2; Developmental Hybridoma Bank), GFAP (1:400; DAKO), Synapsin (1:1000; Calbiochem), NeuN (1:400; Chemicon), Gad65/67 (1:800; Sigma), and Map2 (1:400; Sigma). After washing, Alexa fluor-conjugated secondary antibodies (1:1000; Molecular Probes) were applied for $45 \mathrm{~min}$ at room temperature. Phase and fluorescent images were taken with a Zeiss Axiovert 200 inverted microscope and a Zeiss AxioCam MRm digital camera with AxioVision 4 software.

\section{In utero electroporation and analysis}

Plasmids were transfected in vivo by electroporation (Bai et al. 2003). Briefly, timed pregnant Swiss Webster mice (at 14-14.5 $\mathrm{d}$ of gestation) were anesthetized with isoflourane. The uterine horns were exposed, and 1-1.5 $\mu \mathrm{L}$ (DNA 1-2 $\mu \mathrm{g} / \mu \mathrm{L}$ ) of plasmids in saline with Fast Green $(2 \mathrm{mg} / \mathrm{mL}$; Sigma) were microinjected (Nanonject II; Drummond Scientific) into the lateral ventricles. A $500-\mu \mathrm{F}$ capacitor charged to $50-100 \mathrm{~V}$ was discharged across the embryonic head via copper alloy plates. For cell count analysis, three 16- $\mu \mathrm{m}$-thick coronal cryostat sections from each animal were examined and the cells counted to calculate the average number of $\mathrm{eGFP}^{+}$cells in each region; three different animals were used to generate the averages. Regions were delineated as used previously (Bai et al. 2003; Fasano et al. 2007).

\section{Adult SVZ injections}

Bmi-1 overexpression or EV control $(30,000$ particles in $1 \mu \mathrm{L}$, four animals per condition) were stereotaxically injected into the dorsal horn of the SVZ of 10- to 14-wk-old Swiss Webster mice using coordinates $\mathrm{AP}+0.5 ; \mathrm{ML}+1.1$; from bregma and DV -1.9 from the pial surface. Mice were sacrificed 2 wk following injection, and the CNS tissue was fixed by transcardial perfusion with PBS followed by $4 \%$ paraformaldehyde. To count cells in the olfactory bulb, five different fields at $20 \times$ were used from each animal.

\section{Immunohistochemistry}

Embryonic brains were fixed in 4\% paraformaldehyde (PFA) and frozen. Adult brains were harvested after intracardial perfusion of PBS followed by $4 \%$ PFA. Brains were post-fixed overnight in
$4 \%$ PFA and cryoprotected in $30 \%$ sucrose and frozen. Coronal or sagittal sections were cut on a Leica cryostat at $16 \mu \mathrm{m}$ for embryonic brain and $30 \mu \mathrm{m}$ for adult brain. Sections were blocked in PBS containing 10\% Normal Goat Serum and 0.3\% Triton X. Sections were immunostained with primary antibodies incubated overnight at $4^{\circ} \mathrm{C}$. The antibodies used were Nestin (1:10; Developmental Hybridoma Bank), Ki67 (1:1000; Novocastra), eGFP (1:800; Molecular Probes), Foxg1 (1:200; a gift from Dr. Yoshiki Sasai), Bmi-1 (Upstate Biotechnologies) and monoclonal GFAP (gift from Dr. Harry Kimelberg).

\section{FACS analysis}

The cortices of E14.5 mouse embryos were dissected, dissociated, and plated in culture. At day 0 (D0), the cells were transduced with EV control or Bmi-1-expressing viral vectors. At D7 post-transduction, the cells were sorted for LeX (CD15; Becton Dickenson) as described previously (Sun et al. 2005). After FACS, RNA was isolated from both populations. The FACS experiment was performed twice, and quantitative RT-PCR (qRT-PCR) was carried out in triplicate for both experiments.

\section{Real-time qPCR (RT-qPCR)}

Total RNA was extracted from viral-transduced cells using RNAeasy (Qiagen). cDNA was prepared with SuperScript III (Invitrogen) and used as templates for PCR. PCR core reagents and SYBR green (ABI) were used with $200 \mathrm{nM}$ forward and reverse primers. RT-qPCR was performed with the ABI PRISM7700 Sequence Detection System. A standard curve was generated for each primer pair, and genes of interested were assigned a relative expression value interpolated from this standard curve using the threshold cycle $(\mathrm{Ct})$ according to ABI instructions. All expression values were normalized against GAPDH. All amplifications were done in duplicate, and at least three technical and two biological replicates were performed. PCR primer sequences were used as previously described for Bmi-1 (Fasano et al. 2007).

\section{Western blots}

Protein was isolated using 2\% SDS with EDTA, and protein concentration was calculated using the BCA assay. Equal amounts of protein $(15-20 \mu \mathrm{g} / \mathrm{lane})$ were loaded into a $12 \%$ Invitrogen-NuPAGE Novex BisTris gel and transferred onto nitrocellulose membranes. Membranes were blocked with $5 \%$ milk for $2 \mathrm{~h}$ at room temperature and blotted overnight with antibody at $4^{\circ} \mathrm{C}$ : Bmi-1 (1:500; Upstate Signaling), p21 (1:200; Calbiochem), Foxg1 (1:500; Abcam), and Erk2 (1:5000; Upstate Signaling). Peroxidase-conjugated secondary antibodies (Jackson Immuno Research) were used at $1: 10,000$ for $1 \mathrm{~h}$ at room temperature. ECL (Pierce) was the chemical substrate. Blots were developed with Kodak film.

\section{Statistical analysis}

Results shown are mean \pm SEM. Asterisks and pound signs identify experimental groups that were significantly different from control groups by a one-way ANOVA, with a Bonferroni correction for multiple comparisons ( $P$-value, 0.05$)$, where applicable.

\section{Acknowledgments}

We are grateful to Dr. Yoshiki Sasai for providing the Foxg1 antibody, Dr. Eseng Lai for providing BF1/lacZ-73 ES cells, Dr. Harry Kimelberg for the monoclonal GFAP antibody, Susan 
Goderie and Carol Charniga for valuable technical assistance, and Dr. Qin Shen for helpful discussions. This work was supported by grants R37NS033529 from NINDS (to S.T.) and 5R37DK042989 from NIDDS (to I.R.L.). C.A.F is supported by the New York Stem Cell Foundation, and E.K. is supported by NIDA training grant T32 DA 007307.

\section{References}

Ahlgren, S., Vogt, P., and Bronner-Fraser, M. 2003. Excess FoxG1 causes overgrowth of the neural tube. J. Neurobiol. 57: 337349.

Bai, J., Ramos, R.L., Ackman, J.B., Thomas, A.M., Lee, R.V., and LoTurco, J.J. 2003. RNAi reveals doublecortin is required for radial migration in rat neocortex. Nat. Neurosci. 6: 12771283.

Bruggeman, S.W., Valk-Lingbeek, M.E., van der Stoop, P.P., Jacobs, J.J., Kieboom, K., Tanger, E., Hulsman, D., Leung, C., Arsenijevic, Y., Marino, S., et al. 2007. Ink4a and Arf differentially affect cell proliferation and neural stem cell self-renewal in Bmil-deficient mice. Genes \& Dev. 19: 1438-1443.

Capela, A. and Temple, S. 2002. LeX/ssea-1 is expressed by adult mouse CNS stem cells, identifying them as nonependymal. Neuron 35: 865-875.

Capela, A. and Temple, S. 2006. LeX is expressed by principle progenitor cells in the embryonic nervous system, is secreted into their environment and binds Wnt-1. Dev. Biol. 291: 300313.

Chmielnicki, E., Benraiss, A., Economides, A.N., and Goldman, S.A. 2004. Adenovirally expressed noggin and brain-derived neurotrophic factor cooperate to induce new medium spiny neurons from resident progenitor cells in the adult striatal ventricular zone. J. Neurosci. 24: 2133-2142.

Cui, H., Hu, B., Li, T., Ma, J., Alam, G., Gunning, W.T., and Ding, H.F. 2007. Bmi-1 is essential for the tumorigenicity of neuroblastoma cells. Am. J. Pathol. 170: 1370-1378.

Doetsch, F., Caille, I., Lim, D.A., Garcia-Verdugo, J.M., and Alvarez-Buylla, A. 1999. Subventricular zone astrocytes are neural stem cells in the adult mammalian brain. Cell 97: 703-716.

Dou, C.L., Li, S., and Lai, E. 1999. Dual role of brain factor-1 in regulating growth and patterning of the cerebral hemispheres. Cereb. Cortex 9: 543-550.

Fasano, C.A., Dimos, J.T., Ivanova, N.B., Lowry, N., Lemischka, I.R., and Temple, S. 2007. shRNA knockdown of Bmi-1 reveals a critical role for $\mathrm{p} 21-\mathrm{Rb}$ pathway in NSC selfrenewal during development. Cell Stem Cell 1: 87-100.

Gage, F.H. 2000. Mammalian neural stem cells. Science 287: 1433-1438.

Hatini, V., Tao, W., and Lai, E. 1994. Expression of winged helix genes, BF-1 and BF-2, define adjacent domains within the developing forebrain and retina. J. Neurobiol. 25: 1293-1309.

Irioka, T., Watanabe, K., Mizusawa, H., Mizuseki, K., and Sasai, Y. 2005. Distinct effects of caudalizing factors on regional specification of embryonic stem cell-derived neural precursors. Brain Res. Dev. Brain Res. 154: 63-70.

Ivanova, N., Dobrin, R., Lu, R., Kotenko, I., Levorse, J., DeCoste, C., Schafer, X., Lun, Y., and Lemischka, I.R. 2006. Dissecting self-renewal in stem cells with RNA interference. Nature 442: $533-538$.

Kaplan, M.S. and Bell, D.H. 1984. Mitotic neuroblasts in the 9day-old and 11-month-old rodent hippocampus. J. Neurosci. 4: $1429-1441$.

Kaplan, M.S. and Hinds, J.W. 1977. Neurogenesis in the adult rat: Electron microscopic analysis of light radioautographs. Science 197: 1092-1094.
Leung, C., Lingbeek, M., Shakhova, O., Liu, J., Tanger, E., Saremasiani, P., Van Lohuizen, M., and Marino, S. 2004. Bmil is essential for cerebellar development and is overexpressed in human medulloblastomas. Nature 428: 337-341.

Lois, C. and Alvarez-Buylla, A. 1994. Long-distance neuronal migration in the adult mammalian brain. Science 264: 11451148.

Lois, C., Hong, E.J., Pease, S., Brown, E.J., and Baltimore, D. 2002. Germline transmission and tissue-specific expression of transgenes delivered by lentiviral vectors. Science 295: 868-872.

Lowry, N., Goderie, S.K., Adamo, M., Lederman, P., Charniga, C., Gill, J., Silver, J., and Temple, S 2008. Multipotent embryonic spinal cord stem cells expanded by endothelial factors and Shh/RA promote functional recovery after spinal cord injury. Exp Neurol. 209: 510-522.

Luskin, M.B. 1993. Restricted proliferation and migration of postnatally generated neurons derived from the forebrain subventricular zone. Neuron 11: 173-189.

Marshall, C.A., Suzuki, S.O., and Goldman, J.E. 2003. Gliogenic and neurogenic progenitors of the subventricular zone: Who are they, where did they come from, and where are they going?. Glia 43: 52-61.

Molofsky, A.V., Pardal, R., Iwashita, T., Park, I.K., Clarke, M.F., and Morrison, S.J. 2003. Bmi-1 dependence distinguishes neural stem cell self-renewal from progenitor proliferation. Nature 425: 962-967.

Perrier, A.L., Tabar, V., Barberi, T., Rubio, M.E., Bruses, J.L., Topf, N., Harrison, N.L., and Studer, L. 2004. Derivation of midbrain dopamine neurons from human embryonic stem cells. Proc. Natl. Acad. Sci. 101: 12543-12548.

Piccirillo, S.G., Reynolds, B.A., Zanetti, N., Lamorte, G., Binda, E., Broggi, G., Brem, H., Olivi, A., Dimeco, F., and Vescovi, A.L. 2006. Bone morphogenetic proteins inhibit the tumorigenic potential of human brain tumour-initiating cells. Nature 444: 761-765.

Qian, X., Davis, A.A., Goderie, S.K., and Temple, S. 1997. FGF2 concentration regulates the generation of neurons and glia from multipotent cortical stem cells. Neuron 18: 81-93.

Qian, X., Shen, Q., Goderie, S.K., He, W., Capela, A., Davis, A.A., and Temple, S. 2000. Timing of CNS cell generation: A programmed sequence of neuron and glial cell production from isolated murine cortical stem cells. Neuron 28: 69-80.

Reynolds, B.A. and Rietze, R.L. 2005. Neural stem cells and neurospheres-Re-evaluating the relationship. Nat. Methods 2: 333-336.

Reynolds, B.A. and Weiss, S. 1992. Generation of neurons and astrocytes from isolated cells of the adult mammalian central nervous system. Science 255: 1707-1710.

Seoane, J., Le, H.V., Shen, L., Anderson, S.A., and Massague, J. 2004. Integration of Smad and forkhead pathways in the control of neuroepithelial and glioblastoma cell proliferation. Cell 117: 211-223.

Shen, L., Nam, H.S., Song, P., Moore, H., and Anderson, S.A. 2006. FoxG1 haploinsufficiency results in impaired neurogenesis in the postnatal hippocampus and contextual memory deficits. Hippocampus 16: 875-890.

Shen, Q., Wang, Y., Dimos, J.T., Fasano, C.A., Phoenix, T.N., Lemischka, I.R., Ivanova, N.B., Stifani, S., Morrisey, E.E., and Temple, S. 2006. The timing of cortical neurogenesis is encoded within lineages of individual progenitor cells. Nat. Neurosci. 9: 743-751.

Siegenthaler, J.A. and Miller, M.W. 2005. Transforming growth factor $\beta 1$ promotes cell cycle exit through the cyclin-dependent kinase inhibitor p21 in the developing cerebral cortex. J. Neurosci. 25: 8627-8636. 
Fasano et al.

Sun, Y., Goderie, S.K., and Temple, S. 2005. Asymmetric distribution of EGFR receptor during mitosis generates diverse CNS progenitor cells. Neuron 45: 873-886.

Watanabe, K., Kamiya, D., Nishiyama, A., Katayama, T., Nozaki, S., Kawasaki, H., Watanabe, Y., Mizuseki, K., and Sasai, Y. 2005. Directed differentiation of telencephalic precursors from embryonic stem cells. Nat. Neurosci. 8: 288-296.

Xuan, S., Baptista, C.A., Balas, G., Tao, W., Soares, V.C., and Lai, E. 1995. Winged helix transcription factor BF-1 is essential for the development of the cerebral hemispheres. Neuron 14: $1141-1152$.

Zencak, D., Lingbeek, M., Kostic, C., Tekaya, M., Tanger, E., Hornfeld, D., Jaquet, M., Munier, F.L., Schorderet, D.F., van Lohuizen, M., et al. 2005. Bmil loss produces an increase in astroglial cells and a decrease in neural stem cell population and proliferation. J. Neurosci. 25: 5774-5783.

Zencak, D., Crippa, S.V., Tekaya, M., Tanger, E., Schorderet, D.E., Munier, F.L., van Lohuizen, M., and Arsenijevic, Y. 2006. BMI1 loss delays photoreceptor degeneration in Rd1 mice. Bmil loss and neuroprotection in Rd1 mice. Adv. Exp. Med. Biol. 572: 209-215.

Zhang, R.L., LeTourneau, Y., Gregg, S.R., Wang, Y., Toh, Y., Robin, A.M., Zhang, Z.G., and Chopp, M. 2007. Neuroblast division during migration toward the ischemic striatum: A study of dynamic migratory and proliferative characteristics of neuroblasts from the subventricular zone. I. Neurosci. 27: 3157-3162. 


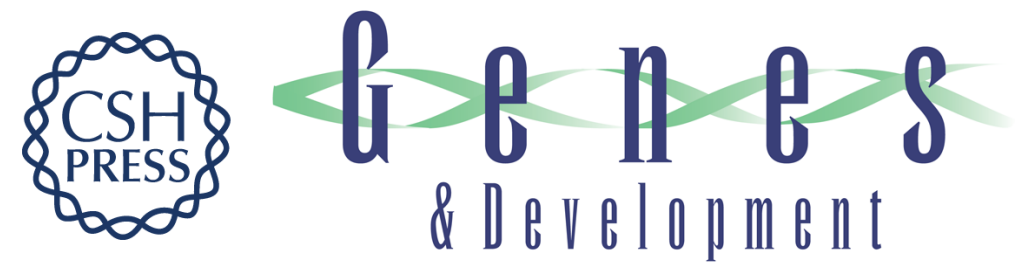

\section{Bmi-1 cooperates with Foxg1 to maintain neural stem cell self-renewal in the forebrain}

Christopher A. Fasano, Timothy N. Phoenix, Erzsebet Kokovay, et al.

Genes Dev. 2009, 23:

Access the most recent version at doi:10.1101/gad.1743709

\section{Supplemental http://genesdev.cshlp.org/content/suppl/2009/03/05/23.5.561.DC1 Material}

References This article cites 39 articles, 11 of which can be accessed free at: http://genesdev.cshlp.org/content/23/5/561.full.html\#ref-list-1

\section{License}

Email Alerting

Receive free email alerts when new articles cite this article - sign up in the box at the top Service 